\title{
Frequency and Associated Costs of Anaphylaxis- and Hypersensitivity-Related Adverse Events for Intravenous Iron Products in the USA: An Analysis Using the US Food and Drug Administration Adverse Event Reporting System
}

\author{
Henry Trumbo ${ }^{1}$ (D) $\cdot$ Karolina Kaluza $^{2} \cdot$ Syed Numan $^{2} \cdot$ Lawrence T. Goodnough $^{3}$
}

Accepted: 10 November 2020 / Published online: 25 November 2020

(c) The Author(s) 2020

\begin{abstract}
Introduction and Objective Intravenous iron preparations rapidly correct iron deficiency anemia, with the notable drug class effect of rare, yet potentially life-threatening, administration-related hypersensitivity or anaphylactic reactions. The objective of this comparative study was to assess adverse events associated with four intravenous iron preparations and estimated medical costs, in the US Food and Drug Administration Adverse Event Reporting System (FAERS) database.

Methods Cases of hypersensitivity reactions and anaphylaxis/anaphylactic shock associated with iron dextran, iron sucrose, ferumoxytol, and ferric carboxymaltose, spontaneously reported to FAERS (1 January, 2014 to 31 December, 2019), were extracted. The reporting odds ratio lower bound $90 \%$ confidence interval (ROR05) $>1$ and cases $\geq 5$ defined a likely signal for a drug-adverse event association. Adverse event-associated medical costs were estimated using Agency for Healthcare Research and Quality/Healthcare Cost and Utilization Project 2016 data.

Results For hypersensitivity reactions, ferumoxytol and iron dextran had the highest ROR05 values (5.00 and 4.35, respectively) and greatest proportions of associated deaths (7.1\% and 5.3\%), followed by iron sucrose (ROR05 3.94, deaths 2.4\%), and ferric carboxymaltose (ROR05 3.03, deaths $0.2 \%$ ). For anaphylaxis/anaphylactic shock, ROR05 for cases/deaths were: $39.32 / 13.4 \%$, ferumoxytol; $37.80 / 4.5 \%$, iron dextran; $17.60 / 4.7 \%$, iron sucrose; and 8.77/no deaths, ferric carboxymaltose. Downstream medical costs per adverse event were highest with iron dextran (US\$8615) and ferumoxytol (US\$8164), followed by iron sucrose (US\$4212), and ferric carboxymaltose (US\$1832).

Conclusions Reporting rates of hypersensitivity and anaphylaxis with intravenous iron preparations were highest with ferumoxytol and lowest with ferric carboxymaltose in the US FAERS database. Adverse event-related medical costs were highest for iron dextran and ferumoxytol, and lowest for ferric carboxymaltose.
\end{abstract}

\section{Introduction}

Iron deficiency is the most frequent cause of anemia, which affects $32.9 \%$ of the global population [1], and of all nutrient deficiencies, it is the only one that is also commonly

Electronic supplementary material The online version of this article (https://doi.org/10.1007/s40264-020-01022-2) contains supplementary material, which is available to authorized users.

Henry Trumbo

htrumbo@stmaryhealthcare.org

1 Pharmacy Department, St. Mary Medical Center, 1201

Langhorne-Newtown Road, Langhorne, PA 19047, USA

2 American Regent, Norristown, PA, USA

3 Stanford University, Stanford, CA, USA detected in industrialized countries [2]. Both iron deficiency and anemia have substantial socioeconomic consequences in terms of reductions in school performance and work productivity [2]. There are numerous causes of iron deficiency anemia [3], but usually the first step in managing iron deficiency is to begin with oral iron supplementation [3-5] because an improved diet alone may not address iron repletion needs [5]. Intravenous (IV) iron preparations are reserved for the treatment of patients who cannot tolerate oral iron, have not responded well to oral iron, or for those patients who would benefit from rapid restoration of iron stores [3-5].

The first IV iron preparation to be used was ferric hydroxide, introduced in the early 1930s, but it was found to be associated with serious toxic reactions related to the immediate systemic release of iron [6]. Consequently, new iron preparations were developed utilizing a carbohydrate shell 


\section{Key Points}

Iron deficiency anemia can be rapidly corrected and managed with intravenous iron products; however, rare hypersensitivity or anaphylactic reactions associated with their administration have occurred.

Reporting rates of hypersensitivity and anaphylactic reactions with four intravenous iron preparations (iron dextran, iron sucrose, ferumoxytol, and ferric carboxymaltose) were assessed based on the US Food and Drug Administration Adverse Event Reporting System database.

There were differential reporting rates of hypersensitivity and anaphylactic reactions for intravenous iron products, with ferric carboxymaltose exhibiting the lowest reporting rate and the lowest downstream medical costs per adverse event.

enclosing an iron core, thereby preventing the immediate release of iron and improving tolerability $[6,7]$. The initial iron saccharide preparations were replaced with high-molecular-weight iron dextran preparations in the 1950s. These latter preparations became the mainstay of IV iron therapy; however, they were associated with rare but serious cases of acute hypersensitivity reactions or anaphylaxis [8]. Since the 1970s, a number of additional iron preparations have become available to replace high-density molecular-weight iron dextran: first low-molecular-weight iron dextran (US approval April 1974), ferric gluconate (US approval February 1999), and iron sucrose (US approval June 2000), and more recently ferumoxytol (US approval June 2009), ferric carboxymaltose (US approval July 2013), and iron isomaltoside (US approval January 2020) [6, 7].

All IV iron preparations share a common structure of an $\mathrm{Fe}^{3+}$ hydroxide particle containing a polynuclear core surrounded by a carbohydrate shell in a colloidal solution [7]. However, the chemistry of the carbohydrate moiety forming the shell and the type and strength of the bonds with the iron core vary, resulting in differences of iron stability within the carbohydrate complex. The lower the stability of the complexes, the faster the systemic release of ferric iron and, in general, the greater the risk of infusion reactions, including serious hypersensitivity and anaphylactoid reactions. The three newest IV iron preparations (ferumoxytol, iron isomaltoside, and ferric carboxymaltose) have more stable carbohydrate shells than the earlier preparations [7]. It is important to determine whether this improved stability has correspondingly reduced the risk of hypersensitivity and anaphylactoid reactions. Although all IV iron preparations are associated with the class risk of serious hypersensitivity reactions and anaphylaxis [4] and black-box warnings for these events have been added to the prescribing information for some IV iron preparations [9, 10], the risk is rare [7, 8]. Given the rarity of these serious reactions, randomized controlled trial evidence and associated meta-analyses have been unable to demonstrate meaningful differences between IV iron preparations [11-13]. Large observational claims database retrospective analyses have suggested these reactions may be more frequent with iron dextran vs newer preparations [4] and with ferumoxytol vs iron sucrose (see Supplementary Table S5 in Wetmore et al. [14]), and pharmacovigilance spontaneous reporting database analyses have suggested higher reporting rates with iron dextran, ferumoxytol, and iron isomaltoside than ferric gluconate or ferric carboxymaltose [8, 15-18]. However, methodological concerns [19-23] and a paucity of longer term data on newer IV iron preparations in many of these publications limit the conclusions that can be reached. Thus, up-to-date analyses are required to further explore signals for potential IV iron-adverse event (AE) associations using established methodologies for analysis of pharmacovigilance spontaneous reporting databases [24, 25].

Here, we analyzed real-world AEs associated with IV iron products spontaneously reported to the US Food and Drug Administration (FDA) Adverse Event Reporting System (FAERS) database [26, 27] using established methodologies [28-31]. Estimated consequent medical costs associated with these AEs were calculated using 2016 Agency for Healthcare Research and Quality (AHRQ)/Healthcare Cost and Utilization Project (HCUP) data [32, 33]. For all analyses, we evaluated IV iron preparations currently approved in the USA for the treatment of iron deficiency anemia, namely iron dextran, iron sucrose, ferumoxytol, and ferric carboxymaltose. The approval date of iron isomaltoside in the USA (January 2020) was too recent for this product to be meaningfully included in the current analysis.

\section{Methods}

\subsection{FAERS Analysis}

\subsubsection{Data Source, Extraction, and Processing}

The FAERS is a nationally centralized and computerized information database broadly used by the FDA and other pharmacovigilance experts for post-marketing drug safety surveillance. The FAERS data are made publicly available as a quarterly download on the FDA's website (FDA 2019) [27], but to obtain accurate insight from the database, extensive data cleansing and normalization was applied. Advera's Evidex ${ }^{\circledR}$ platform [28, 30], using RxFilter ${ }^{\circledR}$ data optimization technology and based upon established algorithms [29], 
was utilized to create a clean, drug-mapped, de-duplicated version of the FAERS data for analysis.

Post-marketing case reports of prespecified AEs associated with branded and generic formulations of four IV iron products: iron dextran, iron sucrose, ferumoxytol, and ferric carboxymaltose (a full list of included products is provided in Table S1 of the Electronic Supplementary Material [ESM]) submitted to FAERS between 1 January, 2014 and 31 December, 2019 were extracted. The AEs of interest for these analyses, defined according to the Medical Dictionary for Regulatory Activities (MedDRA ${ }^{\circledR}$ ) version 22.0 preferred terms (PTs) [34] and Standardized Medical Queries (SMQs) [35], were hypersensitivity reaction individual PTs and the hypersensitivity SMQ and anaphylaxis/anaphylactic shock individual PTs and the anaphylactic reaction SMQ. For SMQ searches, "narrow" scope groupings of PT terms were used. Data validation steps were run to verify that all case reports had completed key identification fields, including the patient identifier, case identifier (each patient could have multiple AE case reports), drug sequence identification, and MedDRA ${ }^{\circledR}$ AE terms [29]. Case reports containing incorrectly completed or missing key identification fields were discarded [29]. The proportion of reports with missing key identification fields was calculated to assess the frequency of discarded reports. Among retained reports, the proportion of missing data for the outcomes field (which contains hospitalization and death information), and for age and sex information, were also calculated. Drug mapping steps were then undertaken before duplicate case reports were removed [29].

Primary suspect cases were defined as case reports where the reporter designated a particular drug as the most likely cause of the given side effect; if the patient was taking other drugs at the time of the AE, those drugs were listed as "concomitant" or "secondary" causes. Total suspect cases were defined as all case reports where the specified drug was mentioned in the case report, including: primary suspect, secondary suspect, concomitant, interacting, or not specified (the "suspect" field in FAERS was not filled in).

\subsubsection{Data Analysis}

Overall number of case reports and primary suspect case counts associated with AEs of interest (hypersensitivity reactions and anaphylaxis/anaphylactic shock) and the proportions of cases associated with hospitalization and/or death related to these AEs were calculated, and disproportionality analyses were conducted for the US region. The reporting odds ratio (ROR) [36] was used to identify the strength of association between each iron preparation and $\mathrm{AE}$ of interest and was chosen over the proportional reporting ratio because the latter measure produces biased estimates of the rate or risk ratio whereas the ROR does not
[31]. The ROR is the pharmacovigilance equivalent of the odds ratio and is defined here as the odds for the $\mathrm{AE}$ of interest occurring among the group of case reports for patients receiving the specific IV iron preparation under investigation divided by the odds for the $\mathrm{AE}$ of interest occurring in the comparison group of case reports for patients receiving any other drug administered by any route, where the odds of the event is the probability that the event will occur divided by the probability that the event does not occur [37]. The limits of the $90 \%$ CI of ROR were obtained via an approximation of the normal distribution as described by Van Puijenbroek et al. [38]. Based on the ROR distribution, the lower bound (ROR05) of the CI was calculated, as it provided 95\% certainty that the true mean of the population was at or above the number reported. There is no universally accepted benchmark regarding the numerical level/threshold at which disproportionality analysis signal detection algorithms identify adverse drug reactions [39, 40], thus we defined values of ROR $05 \geq 1$ and cases $\geq 5$ as a signal of a likely drug-AE association. Our main analysis used primary suspect case counts, but we also performed a sensitivity analysis using total suspect case counts. In addition, we performed further analyses to support our primary analysis, notably stratified analyses by age ( $<65$ vs $\geq 65$ years) and sex, and a calculation of the masking ratio for the ROR05 [41, 42]. A disproportionate signal for a given drug-event pair can be masked because of the presence of a disproportionate signal for another drug with the same event of interest in the same database [41]. According to Maignen et al., we calculated the masking ratio for the ROR05 (MRCI) by dividing the ROR05 using the comparison group for the ROR without all other IV iron preparations by the ROR05 calculated for the main analysis (i.e., using the comparison group for the ROR with all other IV iron preparations); an MRCI substantially $>1$ indicates masking [42].

\section{Costs Analysis}

The methods of Hoffman et al. [43] were used to estimate direct downstream medical costs associated with hypersensitivity reactions and anaphylaxis/anaphylactic shock, and unfavorable patient outcomes for each IV iron preparation. The AE and patient outcome data obtained from FAERS case reports were mapped to AE-specific and associated outcome costs based on the most recently available national inpatient hospitalization and aggregate costs for specific diagnoses and procedures obtained from AHRQ/HCUP 2016 data [32, 33]. The cost of hospital death was based on the average cost of a hospital stay ending in death in 2007, as reported in the analyses of Zhao and Encinosa [44]. No discounting was performed and costs were not adjusted by year. 
Data from HCUP were coded to the International Classification of Diseases, Tenth Revision, Clinical Modification (ICD-10-CM) using BioPortal [45], a repository of biomedical ontologies, and an ICD-10 mapping resource was used to assign ICD-10-CM codes to MedDRA ${ }^{\circledR}$ PTs (Table S2 of the ESM). Preferred Terms that could not be directly assigned by the use of BioPortal were manually mapped using the ICD-10-CM coding manual.

To determine medical cost estimates, the frequency of AEs resulting in hospitalization or death were multiplied by the average hospitalization costs and average cost of a hospital stay ending in death, respectively, for each iron preparation. All costs were inflation adjusted on a yearly basis using the medical care services component of the consumer price index (not seasonally adjusted) for each month of December obtained from the US Bureau of Labor Statistics [46]. These costs were aggregated to determine the total medical costs for each drug. Average costs per AE were calculated by dividing the total medical costs for each preparation by the total number of AEs with available cost mapping reported for each IV iron preparation. Average costs per patient for each preparation were calculated by dividing the total medical costs by the total number of patients receiving each IV iron preparation (the total number was estimated from IQVIA [Durham, NC, USA] IV Iron Landscape Claims Data 2019).

\section{Results}

\subsection{AEs Reported for IV Iron Preparations}

Of a total of 5,027,033 FAERS case reports during the period of study (1 January, 2014 to 31 December, 2019), 95 had missing MedDRA ${ }^{\circledR}$ AE terms, meaning that our primary suspect and total suspect case count analysis had a coverage of $99.998 \%$ of case reports for the overall ROR05 estimates. However, missing data were present to a much greater extent for the outcome field in FAERS (57.2\%), meaning that percentages and ROR05 values for hospitalization and death were based upon a $42.8 \%$ coverage. For our stratified analyses, age was not specified in $47.2 \%$ of cases and sex was not specified in $11.1 \%$ of cases.

Primary suspect case counts and disproportionality associated with each reported AE of interest are summarized in Table 1 and Fig. 1. Each of the four iron preparations had an ROR05 for overall SMQ hypersensitivity reactions that was considered a signal of a likely drug-AE association. The ROR05 was highest for ferumoxytol, followed by iron dextran, iron sucrose, and ferric carboxymaltose (Table 1; Fig. 1). For primary suspect cases resulting in death associated with SMQ hypersensitivity reactions, ferumoxytol and iron sucrose had 14 and 5 deaths, respectively, and had ROR05 values (18.53 and 3.77, respectively) that were considered signals for a likely drug-death association; ferric carboxymaltose had only one reported death (Table 1; Fig. 1). Although the ROR05 for death associated with SMQ hypersensitivity reactions indicated a signal for iron dextran (ROR05 8.01), the absolute number of cases $(n=3)$ fell below the signal detection threshold, likely owing to the low number of overall reports with iron dextran during the timeframe of the study. For primary suspect cases resulting in hospitalization associated with SMQ hypersensitivity reactions, ferric carboxymaltose had the lowest ROR05 value (ROR05 1.2 vs 5.98-9.81 for other IV iron preparations: Table 1; Fig. 1).

All IV iron preparations had an ROR05 for an overall SMQ anaphylactic reaction that was considered a signal of a likely drug-AE association. The ROR05 was highest for ferumoxytol, followed by iron dextran, iron sucrose, and ferric carboxymaltose (Table 1; Fig. 1). For primary suspect cases resulting in death associated with an SMQ anaphylactic reaction, no deaths were reported with ferric carboxymaltose, whereas ferumoxytol had nine deaths and the ROR05 (41.88) represented a signal for a likely drug-death association (Table 1; Fig. 1). Although ROR05 values for death associated with an SMQ anaphylactic reaction were elevated for iron dextran (5.20) and iron sucrose (3.85), the absolute number of cases ( $n=1$ and 2, respectively) fell below the signal detection threshold. For primary suspect cases resulting in hospitalization associated with an SMQ anaphylactic reaction, ferric carboxymaltose had the lowest ROR05 value (ROR05 1.79 vs 17.21-42.47 for other IV iron preparations: Table 1; Fig. 1).

Our sensitivity analysis using total suspect case counts showed very similar ROR05 patterns across the IV iron preparations to those using only primary suspect case counts (Table S3 of the ESM); and ROR05 values for the SMQ anaphylactic reaction, and associated deaths and hospitalizations were lowest with ferric carboxymaltose. The stratified analysis of primary suspect case counts by age showed ROR05 values (with at least five cases in each age group) indicating a likely drug-AE association for an SMQ anaphylactic reaction in both age groups, but ROR05 values were higher among patients aged $\geq 65$ vs $<65$ years across all IV iron preparations; ROR05 values were lowest with ferric carboxymaltose in both age groups (Table S4 of the ESM). ROR05 values for hospitalization due to SMQ hypersensitivity were higher among patients aged $\geq 65$ vs $<65$ years for iron dextran, iron sucrose, and ferumoxytol, and ROR05 values for hospitalization due to an SMQ anaphylactic reaction were higher among patients aged $\geq 65$ vs $<65$ years for iron sucrose and ferumoxytol. The stratified analysis of primary suspect case counts by sex showed that ROR05 values (with at least five cases in both male and female patients) for SMQ hypersensitivity or an SMQ anaphylactic reaction 


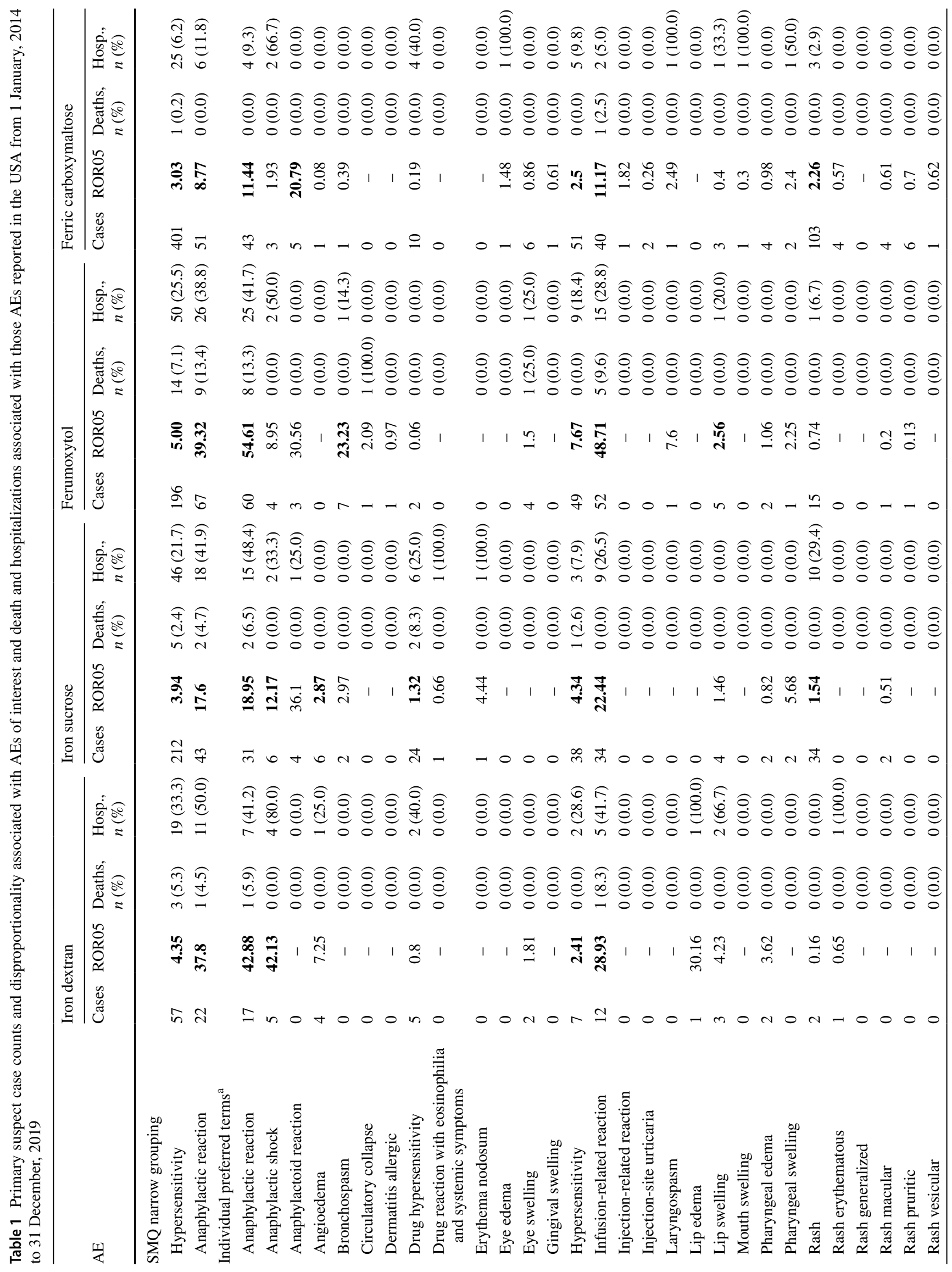




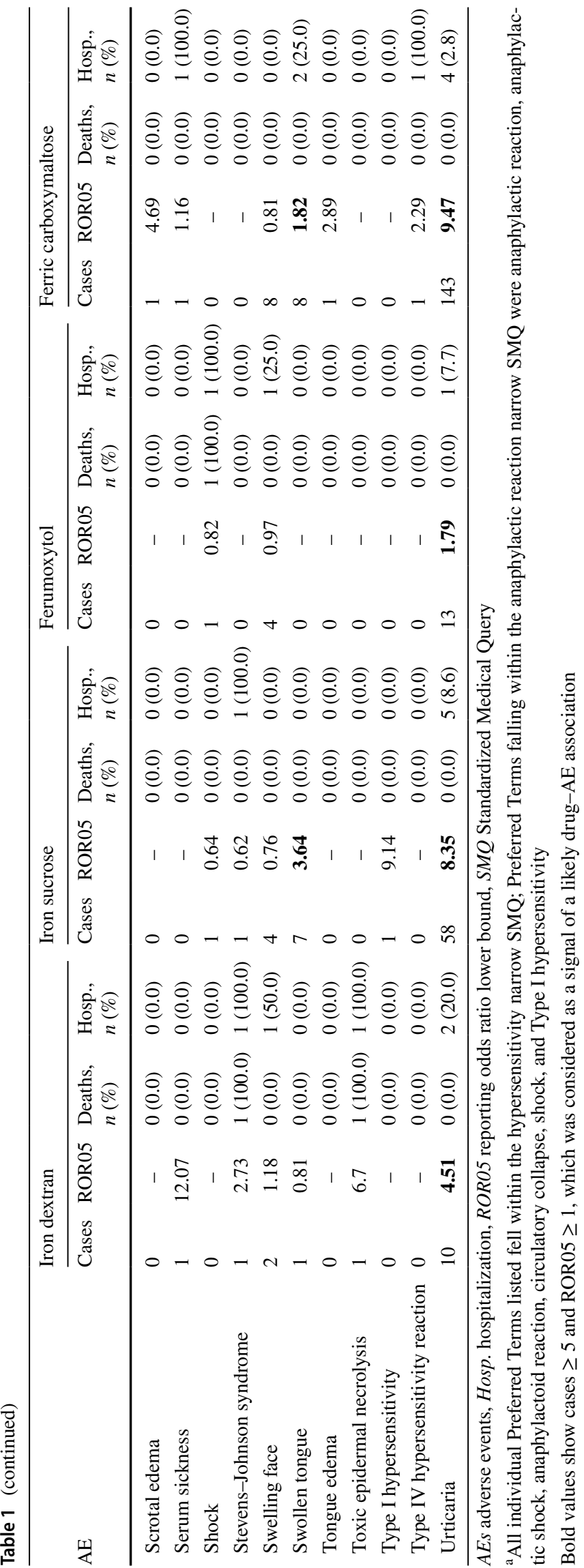

were similarly elevated between male and female patients within each IV iron preparation, but were lowest with ferric carboxymaltose in both male and female patients (Table S4 of the ESM). ROR05 values for hospitalization due to SMQ hypersensitivity or an SMQ anaphylactic reaction were higher among male vs female patients for iron sucrose and ferumoxytol. There were too few deaths in each subgroup to make any meaningful comparison between ROR05 values for deaths by age group or sex. We did not identify any evidence of masking: all MRCI values were close to unity (Table S5 of the ESM).

\section{Medical Costs Associated with Managing AEs of IV Iron Preparations}

A breakdown of costs for each iron preparation is summarized in Table 2 (a breakdown of costs applied to each AE is summarized in Table S6 of the ESM) and total medical cost estimates associated with AEs of iron preparations are summarized in Table 3. For the time period examined, ferumoxytol had the highest total downstream medical costs associated with anaphylaxis and manifestations of hypersensitivity reactions (US\$1,240,963), followed by iron sucrose (US\$724,544), ferric carboxymaltose (US\$650,431), and iron dextran (US\$422,137).

When expressed as average costs for each iron preparation per $\mathrm{AE}$, which accounts for differing reporting rates among the preparations, the highest medical costs per $\mathrm{AE}$ (all hypersensitivity reactions) occurred with iron dextran (US\$8615) and ferumoxytol (US\$8164), followed by iron sucrose (US\$4212) and ferric carboxymaltose (US\$1832). Medical cost estimates associated with anaphylaxis were higher than those associated with hypersensitivity reactions without anaphylaxis (Table 3), with the highest medical costs per anaphylaxis AE occurring with ferumoxytol (US\$9896).

When expressed as average costs for each iron preparation per the estimated total number of patients treated with these preparations, ferumoxytol had the highest average costs (all hypersensitivity reactions) per treated patient (US\$1.24), followed by iron dextran (US\$0.61), ferric carboxymaltose (US\$0.55), and iron sucrose (US\$0.17). The same pattern was evident for average costs per treated patient for hypersensitivity reactions without anaphylaxis and for anaphylaxis (Table 3).

\section{Discussion}

The major finding from this FAERS database study is that there are differential reporting rates of hypersensitivity and anaphylactic reactions for the four studied IV iron products; 


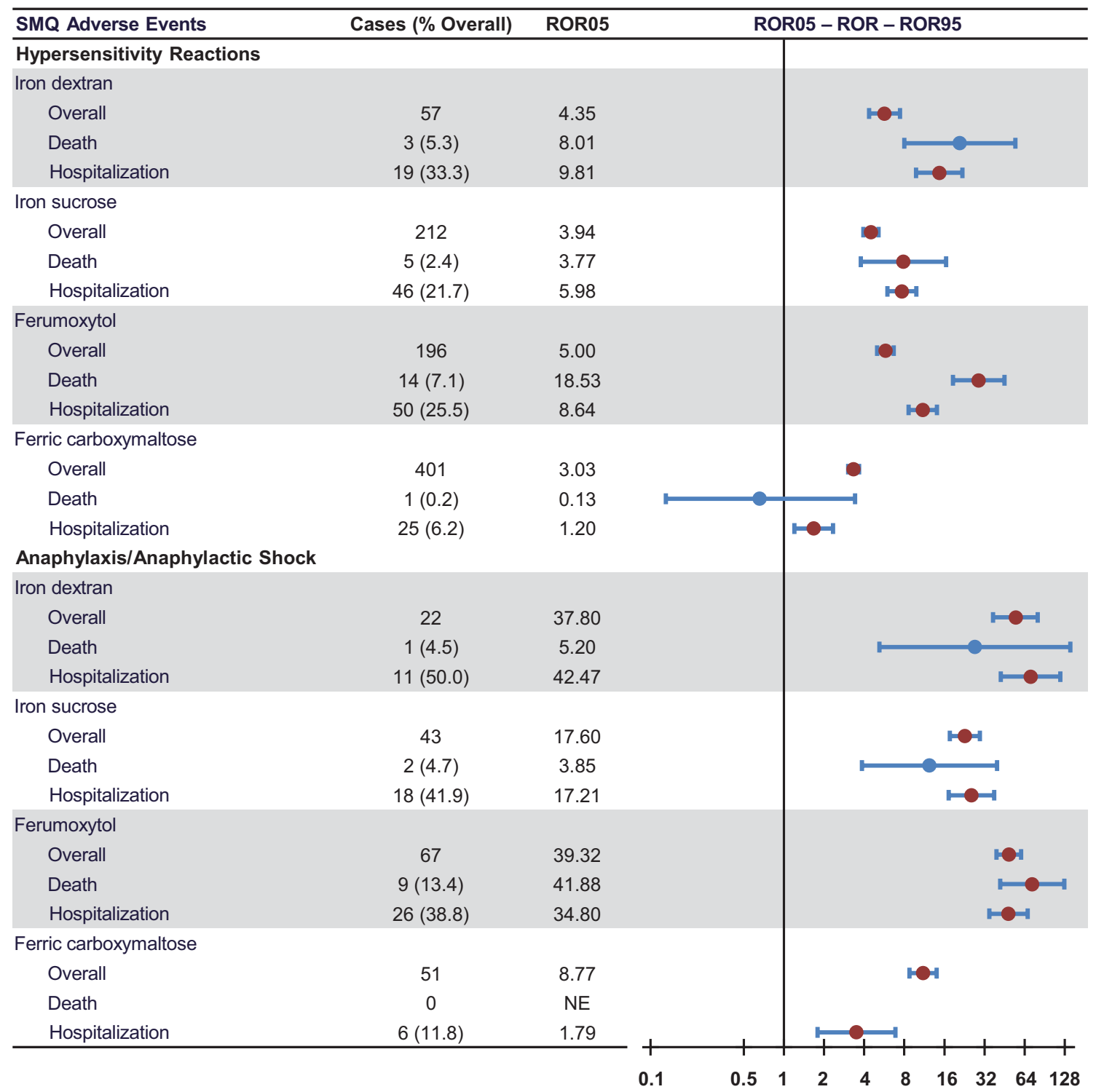

Fig. 1 Primary suspect case counts and disproportionality associated with adverse events (AEs) of interest (Standardized Medical Query [SMQ] terms) and death and hospitalizations associated with those AEs reported in the USA from 1 January, 2014 to 31 December, 2019. Data shown are primary suspect case counts and dispropor-

reporting rates were highest for ferumoxytol and iron dextran, intermediate for iron sucrose, and lowest for ferric carboxymaltose. This finding from the national US spontaneous reporting system, FAERS, is consistent with prior real-world studies, both using spontaneous reporting databases and claims databases $[4,8,15,16]$. In addition, our analysis used the ROR, which is less likely to result in biased estimates than other disproportionality measures [31], as well as providing new cost estimates associated with these AEs. This type of assessment differs from clinical trials, which tionality (reporting odds ratio [ROR] with upper [ROR95] and lower bounds [ROR05] of the $90 \%$ confidence interval) associated with AEs of interest. Events with cases $\geq 5$ and ROR05 $\geq 1$ were considered as a signal for a likely drug- $\mathrm{AE}$ association

are designed to increase the likelihood that drug efficacy signals can be detected. Specifically, most inclusion and exclusion criteria of clinical trials are designed to include a relatively homogenous study population, that may comprise individuals at lower risk of AEs. For example, a head-tohead clinical trial whose primary endpoint was evaluation of safety, and specifically hypersensitivity and anaphylactic reactions, among patients treated with either ferumoxytol or ferric carboxymaltose, excluded patients with a prior history of allergies to drugs that did not include those under 
investigation [12]. This, together with the small sample sizes in most clinical trials, means that uncommon yet clinically important AEs may not be detected before drug approval. Therefore, careful post-approval monitoring is vital to the ongoing safety evaluation of approved therapies. The FDA professionals and pharmacovigilance experts routinely look to the FAERS data for post-marketing drug safety surveillance and as a guide to and signal generator of drug safety issues [43].

Approved IV iron preparations are an important component of current therapeutic armamentariums [2], but are known to be associated with rare and/or serious hypersensitivity reactions and anaphylaxis $[4,5]$. Using a disproportionality analysis of FAERS primary suspect cases, we found that all IV iron preparations were associated with signals for overall hypersensitivity and anaphylactic reactions, but ROR05 values were highest for ferumoxytol and iron dextran followed by iron sucrose, with ferric carboxymaltose having the lowest ROR05 values. Signals for death associated with hypersensitivity and anaphylactic reactions were present for ferumoxytol, whereas only one death associated with a hypersensitivity reaction occurred with ferric carboxymaltose. Signals for hospitalization associated with hypersensitivity and anaphylactic reactions were present for ferumoxytol, iron dextran, and iron sucrose, with ferric carboxymaltose having the lowest ROR05 value. Reporting rates were also lowest with ferric carboxymaltose in various supplementary analyses, including an analysis of total suspect case counts and analyses of primary case counts stratified by age group and sex. In addition, these lower reporting rates with ferric carboxymaltose results could not be explained by masking owing to the presence of other IV iron preparations reporting similar AEs in the same database; MRCI values were all very close to unity.

An alternative assessment of a drug's potential safety risk can be achieved by estimating the magnitude of downstream direct medical costs based on $\mathrm{AE}$ and outcome costing data using the methods of Hoffman et al. [43]. These methods provide an accessible reference point regarding real-world differences in post-marketing drug safety [43]. The results obtained can be used to improve patient safety by identifying therapies that cause an undue burden on patients and healthcare providers. The current analyses showed that over the time period examined, total downstream medical costs associated with anaphylaxis and manifestations of hypersensitivity reactions were highest with ferumoxytol (US\$1,240,963). Total costs were about 42-66\% lower with the three other IV iron preparations. When average costs per $\mathrm{AE}$ were considered, which accounts for differences in $\mathrm{AE}$ reporting rates, iron dextran and ferumoxytol were associated with the highest costs per AE (US\$8615 and US\$8164, respectively). When average costs per treated patient associated with these AEs were considered, ferumoxytol again had the highest cost, followed by iron dextran, ferric carboxymaltose, and iron sucrose. Thus, using the system of Hoffman et al. [43], results suggest that ferumoxytol is associated with the greatest burden of anaphylaxis and manifestations of hypersensitivity reactions, and that ferric carboxymaltose and iron sucrose are associated with the lowest burden of these events. The low burden of events with ferric carboxymaltose is not unexpected because this preparation is characterized by a tight binding of elemental iron to the carbohydrate polymer shell [7] and has the lowest labile-free iron release of the IV iron preparations considered [47].

While real-world studies, both using spontaneous reporting databases and claims databases have shown results consistent with our analysis $[4,8,15,16]$, other analyses have not [11]. In a meta-analysis of randomized controlled trials examining AEs across trials comparing ferumoxytol with other pooled IV iron preparations (iron sucrose and ferric carboxymaltose), oral iron, and placebo, an elevated rate of hypersensitivity reactions and hypotension was noted with ferumoxytol vs oral iron, but not vs the pooled IV iron group. However, only three studies contributed to this latter comparison and all three studies specifically excluded patients with a prior history of allergies to drugs that did not include those under investigation [11]. In an earlier, but much larger, meta-analysis of 103 clinical trials, infusion reactions were increased with all IV iron preparations examined (iron sucrose, ferric gluconate, and ferric carboxymaltose) compared with placebo, oral iron, or no iron, and ferric carboxymaltose was associated with a reduced rate of cardiovascular AEs and AEs leading to discontinuation [13]. However, this analysis did not include ferumoxytol and made no comparisons among the IV iron preparations examined.

\subsection{Limitations}

A number of limitations of our analyses must be acknowledged. These analyses used FAERS data that have wellrecognized limitations: the FDA does not require a causal relationship for an event to be reported; the "primary suspect" designation in FAERS is subjective; many AEs are not reported to FAERS; and reporting rates to FAERS are likely to be low in general, may not be similar across the included drugs, and may vary as a function of drug approval dates. Estimates of the impact of FAERS underreporting vary in the literature, but one study found that roughly $20-33 \%$ of the expected serious events were reported to FAERS for selected biologics and narrow therapeutics index drugs examined [48]. In addition, although reports lodged in FAERS are evaluated by clinical reviewers to monitor the safety of products after they are approved by the FDA, there is no certainty that the reported event was caused by a named product or that all AEs relating to a product are reported 


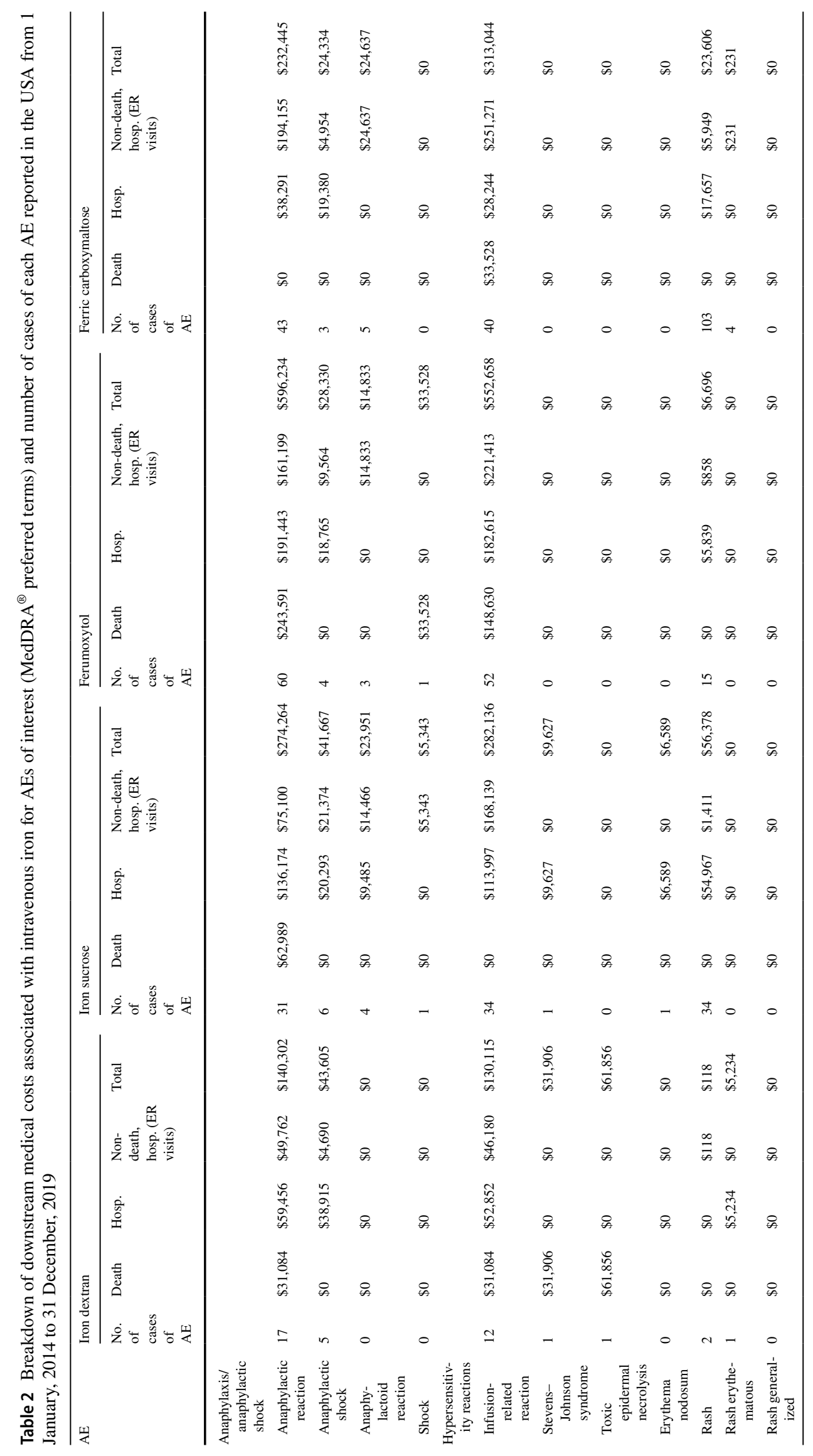




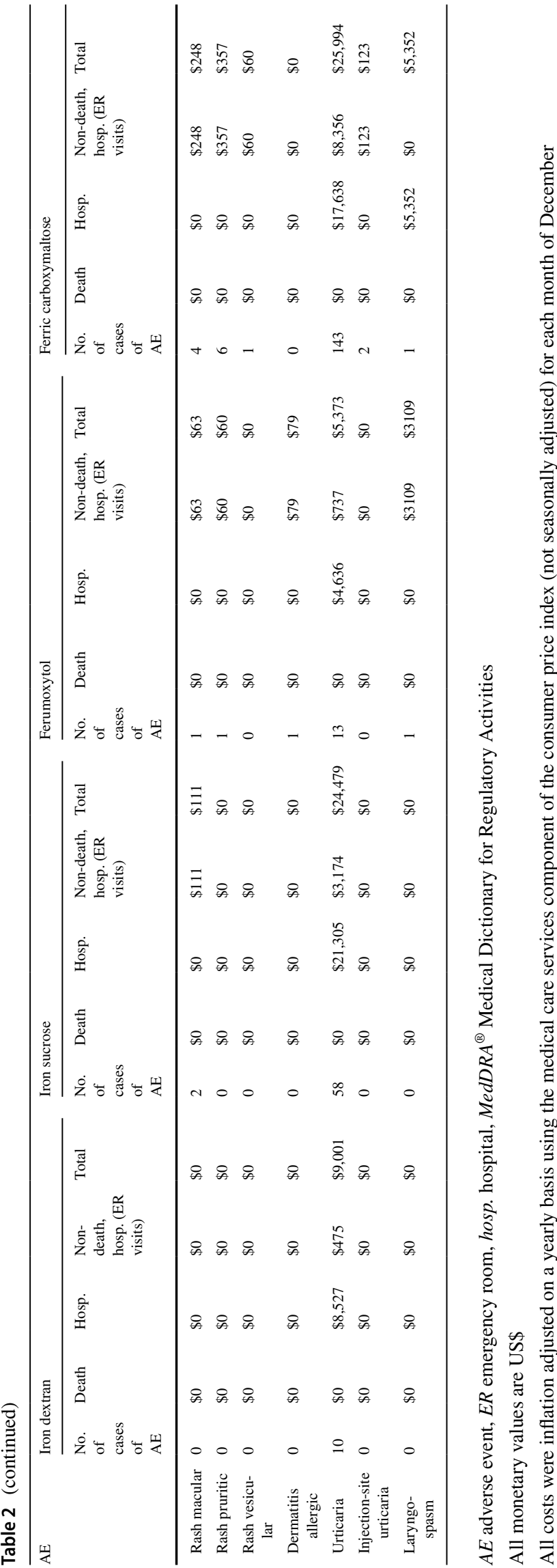

[26]. However, we conducted a sensitivity analysis of total suspect case reports, regardless of attributed causality as in the primary suspect case analysis, and found similar results. Moreover, spontaneous reporting databases suffer from the general limitation that only a numerator is available, which may itself be subject to reporting bias. Therefore, in the absence of denominator information on the overall population exposed to the drug, the specific risk associated with the drug is difficult to estimate. While disproportionality measures aim to overcome this limitation by quantifying to what extent reported AEs are occurring more frequently than expected with a specific drug vs all drugs, they can only indicate differential reporting rates and not actual differences in the occurrence of the event $[24,25,49]$. Thus, spontaneous reporting databases are useful for signal detection [25], whereas other data sources should be used for signal validation or comparative safety-ideally large, adequately powered, randomized controlled trials, or in the case of rare events, large prospectively designed observational studies conducted in representative populations.

Another general limitation of a disproportionality analysis is potential masking, where a disproportionate signal for a given drug-event pair can be masked because of the presence of a disproportionate signal for another drug with the same event of interest in the same database [41]. However, our analysis of MRCI did not show any evidence of masking or dilution of the signal for any of the IV iron preparations examined. An additional general limitation is that information on demographic and pre-existing medical history is limited in spontaneous reporting databases, making adjustment for confounding factors difficult. Age and sex information is available in FAERS, and while this demographic information is limited, our stratified analyses by age group showed that reporting rates of anaphylaxis tended to be higher among patients aged $\geq 65$ vs $<65$ years for all IV iron preparations and reporting rates for hospitalization due to hypersensitivity or anaphylactic reactions with iron sucrose and ferumoxytol were higher among patients aged $\geq 65$ vs $<65$ years and among male vs female patients. Nevertheless, it is important to note that signals indicating a likely drug-AE association also occurred among patients aged $<65$ years and among female patients, meaning that this analysis is relevant to patients of all ages and sex. However, these stratified analyses were limited by missing information on age and sex in $47.2 \%$ and $11.1 \%$ of cases, respectively. In addition, although outcome data are collected in FAERS, this information was missing in a substantial number of reports (e.g., for hospitalization and death in our analysis, coverage was only $42.8 \%$ ); thus, this limitation should be acknowledged when assessing reporting rates for hospitalization and death in this analysis. Other relevant limitations specific to this analysis include the low number of overall reports with iron dextran during the timeframe of 
Table 3 Aggregated medical costs associated with managing AEs of interest for intravenous iron preparations reported in the USA from 1 January 2014 to 31 December, 2019

$$
\begin{array}{cll}
\text { Death costs } \text { Hospital costs } & \begin{array}{l}
\text { Non-death, } \\
\text { hospital costs } \\
\text { (ER visit) }
\end{array}
\end{array}
$$$$
\text { Total costs Total AEs Average costs/AE Total patients Average }
$$

Hypersensitivity

reactions: including

anaphylaxis/anaphy-

lactic shock

Iron dextran

Iron sucrose

Ferumoxytol

Ferric carboxymalt-

ose

Hypersensitivity

reactions: excluding

anaphylaxis/anaphy-

lactic shock

Iron dextran

Iron sucrose

Ferumoxytol

Ferric carboxymalt-

ose

Anaphylaxis/anaphy-

lactic shock

Iron dextran

Iron sucrose

Ferumoxytol

Ferric carboxymalt-

ose

$\begin{array}{lll}\$ 155,929 & \$ 164,983 & \$ 101,225 \\ \$ 62,989 & \$ 372,437 & \$ 289,119 \\ \$ 425,749 & \$ 403,298 & \$ 411,915 \\ \$ 33,528 & \$ 126,562 & \$ 490,341\end{array}$

\section{$\$ 124,845$}

$\$ 0$

$\$ 66,612$

$\$ 206,484$

$\$ 148,630$

$\$ 193,090$

$\$ 68,891$

$\$ 46,773$

$\$ 172,835$

$\$ 226,319$

$\$ 266,596$

$\begin{array}{lr}\$ 31,084 & \$ 98,371 \\ \$ 62,989 & \$ 165,952 \\ \$ 277,120 & \$ 210,208 \\ \$ 0 & \$ 57,671\end{array}$

$\$ 54,453$

$\$ 183,907 \quad 22$

$\$ 345,225 \quad 42$

$\$ 116,283$

$\$ 672,925 \quad 68$

$\$ 281,416 \quad 51$
$\$ 8,615$
$\$ 4,212$
$\$ 8,164$
$\$ 1,832$

695,827

$4,268,967$

$1,004,275$

$1,183,784$

$\$ 0.61$

$\$ 0.17$

$\$ 1.24$

$\$ 0.55$

$A E$ adverse event, $E R$ emergency room

All monetary values are US\$

All costs were inflation adjusted on a yearly basis using the medical care services component of the consumer price index (not seasonally adjusted) for each month of December

the study. It is well known that reporting frequency varies as a function of time since approval, which should be considered when assessing iron dextran results (see Fig. S1 of the ESM). It is also possible that FDA boxed warnings concerning some IV iron preparations may have led to increased reporting.

Findings for the cost estimates may not be comprehensive because they were obtained by mapping AHRQ HCUP cost survey data to MedDRA ${ }^{\circledR}$ terms found in FAERS; not all FAERS hypersensitivity MedDRA ${ }^{\circledR}$ terms had an associated cost mapping available. Thus, potential variations between the FAERS patient population and those used for HCUP surveys could influence estimates. In addition, the most recent available data from the AHRQ HCUP cost survey data were from the year 2016 [32, 33], and for hospital stays resulting in death, the year 2007 [44]. Because of the limited available costing data, we operated on the assumption that the estimated hospitalization costs for the given $\mathrm{AE}$ remained the same year to year; however, we adjusted these calculations for inflation on a yearly basis using the medical care services component of the consumer price index available from the US Bureau of Labor Statistics. We specifically analyzed downstream medical costs only as we wished to focus on costs attributable to AEs not confounded by the acquisition costs of the various IV iron preparations. Finally, patients reporting AEs for each IV iron preparation were not matched on baseline characteristics, thus differences in prescribing patterns could account for some differences in AEs (e.g., one preparation might be used more frequently for patients with comorbidities or severe iron deficiency anemia).

\section{Conclusions}

We found that although all the IV iron formulations we considered had signals for likely drug-AE associations based on ROR05 for hypersensitivity reactions and anaphylaxis, signals were highest for ferumoxytol and iron dextran, 
intermediate for iron sucrose, and lowest for ferric carboxymaltose. When hospitalization and deaths associated with anaphylaxis or hypersensitivity reactions were considered, we found that the signal was highest for ferumoxytol and iron dextran, intermediate for iron sucrose, and lowest for ferric carboxymaltose. Downstream direct medical costs based on these AEs supported these findings. Results of this real-world analysis of FAERS data suggest that reporting rates of anaphylaxis and other hypersensitivity reactions among patients requiring an IV iron preparation are lowest with ferric carboxymaltose.

Acknowledgements Julian Martins and Caroline Spencer of inScience Communications, Springer Healthcare, provided medical writing support funded by American Regent, Inc. The authors thank Julian Martins for his review of the data analytic methods and for his insights concerning interpretation of the data.

\section{Declarations}

Funding This study was supported by American Regent, USA, which holds the marketing rights for ferric carboxymaltose in the USA, and which provided funding to Advera Health Analytics, Inc. (Santa Rosa, CA, USA) to conduct the FAERS data extraction and analysis.

Conflicts of interest/Competing interests Henry Trumbo is a consultant for American Regent, Daiichi Sankyo, and Allergan. Lawrence T. Goodnough is a consultant for American Regent. Karolina Kaluza and Syed Numan are employees of American Regent, the manufacturers of ferric carboxymaltose.

Ethics approval Not applicable.

Consent to participate Not applicable.

Consent for publication Not applicable.

Code availability The code used to conduct this analysis was provided by Evidex ${ }^{\circledR}$ (which is not publicly available), PostgreSQL (a free and open-source relational database), and Microsoft ${ }^{\circledR}$ Excel $^{\circledR}$.

Availability of data and material The source data and analytics for this study were provided by Evidex ${ }^{\circledR}$, licensed by Advera Health Analytics, Inc., and are not publicly available. Healthcare Cost and Utilization Project data are made publicly available by the Agency for Healthcare Research and Quality.

Author contributions HT and LTG contributed to the interpretation of data, and to the reviewing and writing of the report. KK and SN contributed to the conception and design of the analysis, the interpretation of data, and to the reviewing and writing of the report. All authors read and approved the final version.

Open Access This article is licensed under a Creative Commons Attribution-NonCommercial 4.0 International License, which permits any non-commercial use, sharing, adaptation, distribution and reproduction in any medium or format, as long as you give appropriate credit to the original author(s) and the source, provide a link to the Creative Commons licence, and indicate if changes were made. The images or other third party material in this article are included in the article's Creative Commons licence, unless indicated otherwise in a credit line to the material. If material is not included in the article's Creative Commons licence and your intended use is not permitted by statutory regulation or exceeds the permitted use, you will need to obtain permission directly from the copyright holder. To view a copy of this licence, visit http://creativecommons.org/licenses/by-nc/4.0/.

\section{References}

1. Kassebaum NJ, Jasrasaria R, Naghavi M, Wulf SK, Johns N, Lozano R, et al. A systematic analysis of global anemia burden from 1990 to 2010. Blood. 2014;123(5):615-24.

2. World Health Organization. Micronutrient deficiences: iron deficiency anemia. https://www.who.int/health-topics/anaemia. Accessed 20 Apr 2020.

3. Short MW, Domagalski JE. Iron deficiency anemia: evaluation and management. Am Fam Physician. 2013;87(2):98-104.

4. Wang C, Graham DJ, Kane RC, Xie D, Wernecke M, Levenson M, et al. Comparative risk of anaphylactic reactions associated with intravenous iron products. JAMA. 2015;314(19):2062.

5. Clenin GE. The treatment of iron deficiency without anaemia (in otherwise healthy persons). Swiss Med Wkly. 2017;147:w14434. https://doi.org/10.4414/smw.2017.14434.

6. Auerbach M, Ballard H. Clinical use of intravenous iron: administration, efficacy, and safety. Hematology. 2010;2010(1):338-47.

7. Girelli D, Ugolini S, Busti F, Marchi G, Castagna A. Modern iron replacement therapy: clinical and pathophysiological insights. Int J Hematol. 2018;107(1):16-30.

8. Chertow GM, Mason PD, Vaage-Nilsen O, Ahlmen J. Update on adverse drug events associated with parenteral iron. Nephrol Dial Transplant. 2006;21(2):378-82. https://doi.org/10.1093/ndt/gfi25 3.

9. AMAG Pharmaceuticals Inc. Feraheme ${ }^{\circledR}$ (ferumoxytol injection), for intravenous use, prescribing information. Waltham, MA. https://www.accessdata.fda.gov/drugsatfda_docs/label /2018/022180s009lbl.pdf. Accessed 10 Dec 2019.

10. Patheon Italia S.p.A. InFeD ${ }^{\circledR}$ (iron dextran injection USP) prescribing information. https://www.accessdata.fda.gov/drugsatfda _docs/label/2009/017441s171lbl.pdf. Accessed 20 Apr 2020.

11. Abdulrehman J, Tang GH, Auerbach M, Santesso N, Sholzberg $M$. The safety and efficacy of ferumoxytol in the treatment of iron deficiency: a systematic review and meta-analysis. Transfusion. 2019;59(12):3646-56. https://doi.org/10.1111/trf.15587.

12. Adkinson NF, Strauss WE, Macdougall IC, Bernard KE, Auerbach M, Kaper RF, et al. Comparative safety of intravenous ferumoxytol versus ferric carboxymaltose in iron deficiency anemia: a randomized trial. Am J Hematol. 2018;93(5):683-90. https://doi.org/10.1002/ajh.25060.

13. Avni T, Bieber A, Grossman A, Green H, Leibovici L, GafterGvili A. The safety of intravenous iron preparations: systematic review and meta-analysis. Mayo Clin Proc. 2015;90(1):12-23. https://doi.org/10.1016/j.mayocp.2014.10.007.

14. Wetmore JB, Weinhandl ED, Zhou J, Gilbertson DT. Relative incidence of acute adverse events with ferumoxytol compared to other intravenous iron compounds: a matched cohort study. PLoS ONE. 2017;12(1):e0171098. https://doi.org/10.1371/journal.pone.01710 98.

15. Bailie GR, Clark JA, Lane CE, Lane PL. Hypersensitivity reactions and deaths associated with intravenous iron preparations. Nephrol Dial Transplant. 2005;20(7):1443-9. https://doi. org/10.1093/ndt/gfh820.

16. Bailie GR. Comparison of rates of reported adverse events associated with i.v. iron products in the United States. Am J Health Syst Pharm. 2012;69(4):310-20. https://doi.org/10.2146/ajhp110262. 
17. Ehlken B, Nathell L, Gohlke A, Bocuk D, Toussi M, Wohlfeil S. Evaluation of the reported rates of severe hypersensitivity reactions associated with ferric carboxymaltose and iron (III) isomaltoside 1000 in Europe based on data from EudraVigilance and VigiBase between 2014 and 2017. Drug Saf. 2019a;42(3):463-71. https://doi.org/10.1007/s40264-018-0769-5.

18. Nathell L, Gohlke A, Wohlfeil S. Reported severe hypersensitivity reactions after intravenous iron administration in the European Economic Area (EEA) before and after implementation of risk minimization measures. Drug Saf. 2020;43(1):35-43. https://doi.org/10.1007/s40264-019-00868-5.

19. DeLoughery TG, Auerbach M. Is low-molecular weight iron dextran really the most risky iron? Unconvincing data from an unconvincing study. Am J Hematol. 2016;91(5):451-2. https:// doi.org/10.1002/ajh.24326.

20. Ehlken B, Nathell L, Gohlke A, Bocuk D, Toussi M, Wohlfeil S. Authors' reply to Schaffalitzky de Muckadell and colleague's comment on "Evaluation of the Reported Rates of Severe Hypersensitivity Reactions Associated with Ferric Carboxymaltose and Iron (III) Isomaltoside 1000 in Europe Based on Data from EudraVigilance and VigiBase between 2014 and 2017." Drug Saf. 2019b;42(5):693-6. https://doi.org/10.1007/s40264-019-00815-4.

21. Schaffalizky de Muckadell P, Strom CC. Comment on "Evaluation of the Reported Rates of Severe Hypersensitivity Reactions Associated with Ferric Carboxymaltose and Iron(III) Isomaltoside 1000 in Europe Based on Data from EudraVigilance and VigiBase Between 2014 and 2017.” Drug Saf. 2019;42(5):689-91. https://doi.org/10.1007/s40264-019-00814-5.

22. Schaffalizky de Muckadell P, Strom CC. Comment on "Reported Severe Hypersensitivity Reactions after Intravenous Iron Administration in the European Economic Area (EEA) Before and After Implementation of Risk Minimization Measures.” Drug Saf. 2020;43(5):503-5. https://doi.org/10.1007/s40264-020-00929-0.

23. Wysowski DK, Swartz L, Borders-Hemphill BV, Goulding MR, Dormitzer C. Use of parenteral iron products and serious anaphylactic-type reactions. Am J Hematol. 2010;85(9):650-4. https:// doi.org/10.1002/ajh.21794.

24. Bihan K, Lebrun-Vignes B, Funck-Brentano C, Salem JE. Uses of pharmacovigilance databases: an overview. Therapie. 2020;S0040-5957(20):30047. https://doi.org/10.1016/j.thera p.2020.02.022.

25. Sultana J, Trifiro G. The potential role of big data in the detection of adverse drug reactions. Expert Rev Clin Pharmacol. 2020;13(3):201-4. https://doi.org/10.1080/17512433.2020.17400 86.

26. US Food and Drug Administration. FDA Adverse Event Reporting System (FAERS) quarterly data extract files. https://fis.fda.gov/ extensions/FPD-QDE-FAERS/FPD-QDE-FAERS.html. Accessed 7 Apr 2020.

27. US Food and Drug Administration. Questions and answers on FDA's Adverse Event Reporting System (FAERS). 2019. https ://www.fda.gov/drugs/surveillance/questions-and-answers-fdasadverse-event-reporting-system-faers. Accessed 7 Apr 2020.

28. Advera Health Analytics Inc. Evidex ${ }^{\circledR}$. https://www.adveraheal th.com/solutions/overview\#software. Accessed 20 Apr 2020.

29. Banda JM, Evans L, Vanguri RS, Tatonetti NP, Ryan PB, Shah $\mathrm{NH}$. A curated and standardized adverse drug event resource to accelerate drug safety research. Sci Data. 2016;3:160026. https://doi.org/10.1038/sdata.2016.26.

30. Hoffman KB, Dimbil M, Tatonetti NP, Kyle RF. A pharmacovigilance signaling system based on FDA regulatory action and postmarketing adverse event reports. Drug Saf. 2016;39(6):561-75.

31. Rothman KJ, Lanes S, Sacks ST. The reporting odds ratio and its advantages over the proportional reporting ratio. Pharmacoepidemiol Drug Saf. 2004;13(8):519-23. https://doi.org/10.1002/ pds.1001.
32. Agency for Healthcare Research and Quality. Healthcare Cost and Utilization Project (HCUP). https://www.ahrq.gov/data/hcup/ index.html. Accessed 20 Apr 2020.

33. Healthcare Cost and Utilization Project (HCUP).https://www. hcup-us.ahrq.gov/. Accessed 20 Apr 2020.

34. Medical Dictionary for Regulatory Activities. MedDRA version 22.0. https://www.meddra.org. Accessed 7 Apr 2020.

35. Medical Dictionary for Regulatory Activities. Standardised MedDRA queries (SMQs). https://www.meddra.org/how-to-use/tools/ smqs. Accessed 7 Apr 2020.

36. Bates A, Evans SJW. Quantitative signal detection using spontaneous ADR reporting. Pharmacoepidemiol Drug Saf. 2009;18(6):427-36.

37. Böhm R. Primer on disproportionality analysis. 2018. http://openv igil.sourceforge.net/doc/DPA.pdf. Accessed 7 Apr 2020.

38. van Puijenbroek EP, Bate A, Leufkens HGM, Lindquist M, Orre R, Egberts ACG. A comparison of measures of disproportionality for signal detection in spontaneous reporting systems for adverse drug reactions. Pharmacoepidemiol Drug Saf. 2002;11(1):3-10.

39. Harpaz R, DuMouchel W, LePendu P, Bauer-Mehren A, Ryan $\mathrm{P}$, Shah NH. Performance of pharmacovigilance signal-detection algorithms for the FDA adverse event reporting system. Clin Pharmacol Ther. 2013;93(6):539-46. https://doi.org/10.1038/ clpt.2013.24.

40. Candore G, Juhlin K, Manlik K, Thakrar B, Quarcoo N, Seabroke $\mathrm{S}$, et al. Comparison of statistical signal detection methods within and across spontaneous reporting databases. Drug Saf. 2015;38(6):577-87. https://doi.org/10.1007/s40264-015-0289-5.

41. Maignen F, Hauben M, Hung E, Van Holle L, Dogne JM. Assessing the extent and impact of the masking effect of disproportionality analyses on two spontaneous reporting systems databases. Pharmacoepidemiol Drug Saf. 2014;23(2):195-207. https://doi. org/10.1002/pds.3529.

42. Maignen F, Hauben M, Dogne JM. A mathematical framework to quantify the masking effect associated with the confidence intervals of measures of disproportionality. Ther Adv Drug Saf. 2017;8(7):231-44. https://doi.org/10.1177/2042098617704143.

43. Hoffman KB, Dimbil M, Kyle RF, Tatonetti NP, Erdman CB, Demakas A, et al. A drug safety rating system based on postmarketing costs associated with adverse events and patient outcomes. J Manag Care Spec Pharm. 2015;21(12):1134-43.

44. Zhao Y, Encinosa W. The cost of end-of-life hospitalizations: Healthcare Cost and Utilization Project (HCUP) statistical brief. 2007. https://www.hcup-us.ahrq.gov/reports/statbriefs/sb81.pdf. Accessed 20 Apr 2020.

45. BioPortal. Mappings. http://bioportal.bioontology.org/mappings. Accessed 7 Apr 2020.

46. US Bureau of Labor Statistics. Consumer price index. 2020. https ://www.bls.gov/bls/news-release/cpi.htm. Accessed 13 Oct 2020.

47. Neiser S, Rentsch D, Dippon U, Kappler A, Weidler PG, Göttlicher J, et al. Physico-chemical properties of the new generation IV iron preparations ferumoxytol, iron isomaltoside 1000 and ferric carboxymaltose. Biometals. 2015;28(4):615-35.

48. Alatawi YM, Hansen RA. Empirical estimation of under-reporting in the U.S. Food and Drug Administration Adverse Event Reporting System (FAERS). Expert Opin Drug Saf. 2017;16(7):761-7. https://doi.org/10.1080/14740338.2017.1323867.

49. Moore N, Hall G, Sturkenboom M, Mann R, Lagnaoui R, Begaud B. Biases affecting the proportional reporting ratio (PPR) in spontaneous reports pharmacovigilance databases: the example of sertindole. Pharmacoepidemiol Drug Saf. 2003;12(4):271-81. https://doi.org/10.1002/pds.848. 